\title{
Comparison of oral anticoagulation use and adherence among Medicare beneficiaries enrolled in stand-alone prescription drug plans vs Medicare Advantage prescription drug plans
}

\author{
Terri Victoria Newman, PharmD, MS; Nico Gabriel, MA; Qinfeng Liang, MS; Coleman Drake, PhD; \\ Samar R El Khoudary, MPH, PhD; Chester B Good, MD, MPH; Walid F Gellad, MD, MPH; \\ and Inmaculada Hernandez, PharmD, PhD
}

\section{What is already known about this subject}

- Oral anticoagulants (OACs) are beneficial in reducing stroke events in atrial fibrillation (AF) patients but are widely underused by patients recommended for OAC treatment.

- To mitigate OAC underuse, experts from academia, government, and industry propose the implementation of payment models that align health system financial incentives to improve OAC use.

- Limited evidence suggests that Medicare Advantage prescription drug (MAPD) plans, which integrate medical and pharmacy benefits, can incentivize the use of medications with clinical benefit, such as OACs, yet this has not been evaluated in $\mathrm{AF}$ populations.

\section{What this study adds}

- A retrospective analysis of Medicare claims data explored how different Part D insurance designs can affect the use of OACs among an AF population.

- Beneficiaries enrolled in MAPD plans, which bear financial risk for medical and pharmacy costs, used and adhered to OACs more frequently than beneficiaries enrolled in stand-alone prescription drug plans.

\section{ABSTRACT}

BACKGROUND: For atrial fibrillation (AF) patients, oral anticoagulants (OACs) can reduce the risk of stroke by $60 \%$; however, nearly $50 \%$ of patients recommended to receive OACs do not receive therapy. Integrated insurers that cover pharmacy and medical benefits may be incentivized to improve OAC use and adherence because they benefit from offsets in medical costs associated with prevented strokes.
OBJECTIVE: To compare OAC use and adherence between $A F$ patients enrolled in Medicare stand-alone prescription drug plans (PDPs), which only cover pharmacy benefits, and those enrolled in Medicare Advantage prescription drug (MAPD) plans, which cover medical and pharmacy benefits.

METHODS: This was a retrospective cohort study, conducted using 2014-2016 Medicare claims data from the Centers for Medicare \& Medicaid Services and a large regional health plan in Pennsylvania. Primary

\section{Author affiliations}

Terri Victoria Newman, PharmD, MS, Department of Pharmacy and Therapeutics, University of Pittsburgh School of Pharmacy, Pittsburgh, PA. Qinfeng Liang, MS, Center for Value-Based Pharmacy Initiatives and High-Value Care, UPMC Health Plan Insurance Services Division, Pittsburgh, PA. Coleman Drake, PhD, Department of Health Policy and Management, University of Pittsburgh Graduate School of Public Health, Pittsburgh, PA. Samar R El Khoudary, MPH, PhD, Epidemiology Data Center, University of Pittsburgh, Pittsburgh, PA. Chester B Good, MD, MPH, Center for Value-Based Pharmacy Initiatives and High-Value Care, UPMC Health Plan Insurance Services Division, and University of Pittsburgh Division of General Internal Medicine, Pittsburgh, PA. Walid F Gellad, MD, MPH, Department of Medicine, University of Pittsburgh, and Center for Health Equity Research and Promotion, VA Pittsburgh Healthcare System, Pittsburgh, PA. Inmaculada Hernandez, PharmD, PhD, and Nico Gabriel, MA, Department of Clinical Pharmacy, University of California, San Diego.

\section{AUTHOR CORRESPONDENCE:}

Terri Victoria Newman, tvn6@pitt.edu

J Manag Care Spec Pharm 2022;28(2):266-74

Copyright $($ C2022, Academy of Managed Care Pharmacy. All rights reserved. 
outcomes included OAC use and OAC adherence. OAC use was measured as filling at least 1 prescription for an OAC after AF diagnosis. OAC adherence was defined as having greater than or equal to $80 \%$ of days covered with an OAC. We constructed conditional logistic regression models in propensity score-matched samples to test the association between enrollment in PDPs or MAPD plans and outcomes.

RESULTS: There were 2,551 AF patients enrolled in PDPs and 4,502 in MAPD plans before propensity score matching. The propensity score-matched sample included 2,537 patients in each group. OAC use was higher among MAPD beneficiaries (74\%-76\%) compared with PDP beneficiaries $(70 \% ; P<0.001)$, and $41 \%-42 \%$ of MAPD beneficiaries were adherent to OACs, compared with $34 \%$ of PDP beneficiaries $(P<0.001)$. In adjusted analyses among propensity score-matched samples, PDP enrollment was associated with lower odds of OAC use $(\mathrm{OR}=0.67,95 \% \mathrm{Cl}=0.56-0.81)$ and adherence $(\mathrm{OR}=0.68,95 \% \mathrm{Cl}=0.59$ 0.78) compared with MAPD enrollment.

CONCLUSIONS: AF patients enrolled in MAPD plans were more likely to use and adhere to OACs compared with PDP enrollees. These results may reflect the financial incentives of MAPD plans to improve guideline-recommended OAC use, since MAPD insurers bear the risk of pharmacy and medical costs and thus may benefit from cost savings associated with averted stroke events. As efforts to improve use and adherence of OACs in AF patients increase, focus should be given to how insurance benefit designs can affect medication use.

Atrial fibrillation (AF) is a highly prevalent cardiac arrhythmia affecting nearly 6 million people in the United States. ${ }^{1-4}$ It is associated with a 5-fold increase in the risk of stroke and accounts for $15 \%-20 \%$ of all ischemic strokes. ${ }^{5}$ Oral anticoagulants (OACs) are a mainstay of treatment for stroke prevention in $\mathrm{AF}$ and reduce stroke risk by $60 \%{ }^{6,7}$ Despite the benefit of stroke prevention, nearly $50 \%$ of AF patients with indications to receive OAC medication do not use this therapy. ${ }^{8}$ Furthermore, among those who initiate OACs, less than $50 \%$ adhere to therapy over time. ${ }^{9}$

OAC therapy has strong cost-saving potential due to averted stroke events. For this reason, experts have proposed the implementation of payment models that align health system financial incentives as one of the top strategies to mitigate $\mathrm{OAC}$ underuse. ${ }^{10,11}$ Medicare beneficiaries, who account for a majority of the AF population, have 2 options for the coverage of pharmacy benefits: they can enroll in stand-alone prescription drug plans (PDPs), which only cover pharmacy services, or they can enroll in Medicare Advantage prescription drug (MAPD) plans, which provide medical and pharmacy coverage.

MAPD plans represent carve-in plans where medical and pharmacy benefits are integrated. PDPs are carve-out plans, where pharmacy benefits are provided by a different payer than medical benefits, which are covered by traditional Medicare. There is evidence to suggest that MAPD plans incentivize the use of medications that have proven clinical benefit, which can potentially offset downstream medical costs; however, there is a paucity of evidence that has evaluated the effect of different Part D insurance designs on OAC use and adherence in the AF population..$^{12,13}$

In this study, we compared OAC use and adherence between PDP and MAPD beneficiaries. We hypothesized that MAPD beneficiaries will be more likely to use and adhere to OACs compared with PDP beneficiaries because, unlike PDPs, MAPD plans benefit from cost savings associated with averted stroke events.

\section{Methods}

\section{DATA SOURCE AND STUDY POPULATION}

We conducted a retrospective cohort study using 2014-2016 deidentified claims data from Medicare fee-for-service beneficiaries enrolled in PDPs through the Centers for Medicare \& Medicaid Services (CMS) and beneficiaries enrolled in MAPD plans provided by a large regional health plan based in Pennsylvania. We used data from this health plan to capture MAPD beneficiaries because CMS data do not contain complete medical information for MAPD beneficiaries, so it was not possible to execute our study solely on the basis of CMS data.

We selected our study population across both cohorts in the following steps (Figures 1 and 2). First, we selected beneficiaries located within Pennsylvania, where the MAPD health plan has the highest market share, with a diagnosis of $\mathrm{AF}$ in 2015-defined as 1 inpatient or 2 outpatient claims with an AF diagnosis in the first or second position (International Classification of Diseases, Ninth/Tenth Revision, Clinical Modification [ICD-9/10-CM] codes are shown in Supplementary Table 1 in the online article). The first day in 2015 that a patient met the definition of AF diagnosis was defined as our index date. Second, because direct oral anticoagulants (DOACs) are not recommended for valvular disease ${ }^{14}$ we excluded any patients with a diagnosis of valvular atrial fibrillation in 2015 (Supplementary Table 1). Third, we restricted the sample to those continuously enrolled for 12 months before the index date, in order to have complete data to define covariates. We further limited the sample to beneficiaries who were continuously enrolled for 12 months after the index date to ensure that we observed their prescription claims. Finally, we excluded beneficiaries with a $\mathrm{CHA}_{2} \mathrm{DS}_{2}$-VASc score less than 2 because, in the years of the study period, professional 


\section{FIGURE 1 Sample Selection: PDP Cohort}

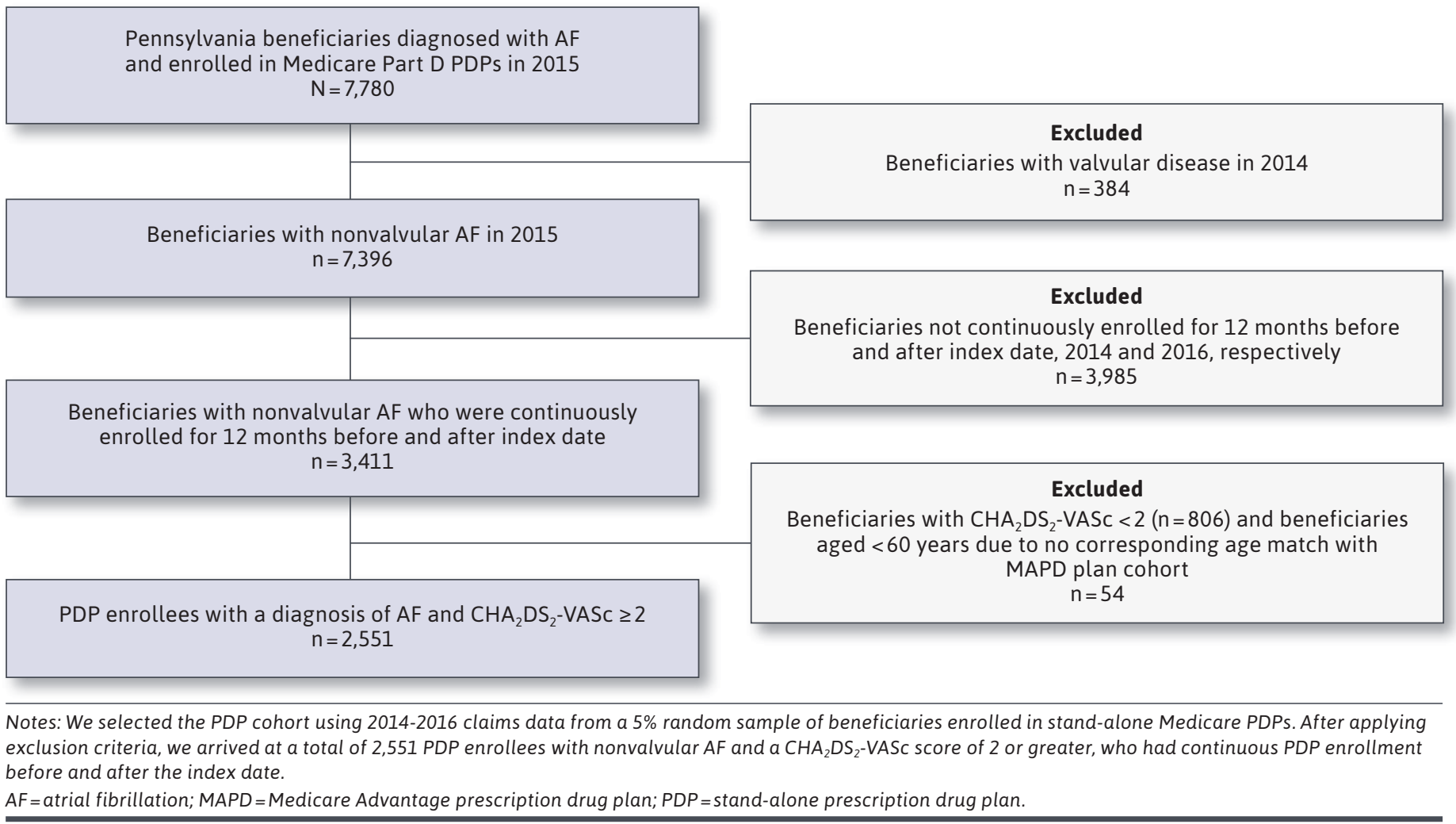

society guidelines recommended $\mathrm{OAC}$ use in $\mathrm{AF}$ patients with a $\mathrm{CHA}_{2} \mathrm{DS}_{2}$-VASc score of 2 or greater. ${ }^{15}$ The $\mathrm{CHA}_{2} \mathrm{DS}_{2}{ }^{-}$ VASc score is a risk prediction tool used to assess the risk of stroke in AF patients based on 5 factors: congestive heart failure (CHF), hypertension, age, diabetes, and previous history of stroke.

\section{INDEPENDENT VARIABLES}

The primary independent variable was the type of Medicare Part D coverage, PDPs vs MAPD plans. PDPs reflect Medicare Part D stand-alone plans that provide pharmacy coverage, whereas MAPD plans provide medical and pharmacy coverage.

Covariates included a comprehensive set of demographic and clinical characteristics. Demographic characteristics included a binary indicator for sex and discrete categories for age $(>80$ or $<80)$. Clinical covariates included previous history of CHF, hypertension, acute myocardial infarction, renal disease, liver disease, diabetes, stroke or transient ischemic attack, intracranial hemorrhage, and major bleeding; previous use of nonsteroidal anti-inflammatory drugs (NSAIDs), antiplatelet drugs, or anticoagulants; and $\mathrm{CHA}_{2} \mathrm{DS}_{2}$-VASc score and HAS-BLED score. Chronic diseases and history of bleeding were measured in the year before the index date using ICD-9/10-CM codes (Supplementary Table 1).

Previous medication use was measured in the 6 months before the index date. $\mathrm{CHA}_{2} \mathrm{DS}_{2}$-VASc scores were calculated using age, sex, history of CHF, hypertension, diabetes, vascular disease, and stroke or transient ischemic attack. ${ }^{14}$ The HAS-BLED score was calculated as the sum of 8 factors: hypertension, abnormal renal or liver function, stroke, history of bleeding, international normalized ratio (INR), age 65 years or older, concomitant use of antiplatelets or NSAIDs, and excess alcohol use. Since Medicare claims data do not contain information on INR levels, we calculated the HASBLED score as the sum of all factors except for labile INR.

\section{OUTCOMES}

We evaluated 2 primary outcomes: OAC use and OAC adherence. OAC use was defined as having at least 1 prescription for any OAC after the index date. As previously defined in 


\section{FIGURE 2 Sample Selection: MAPD Cohort}

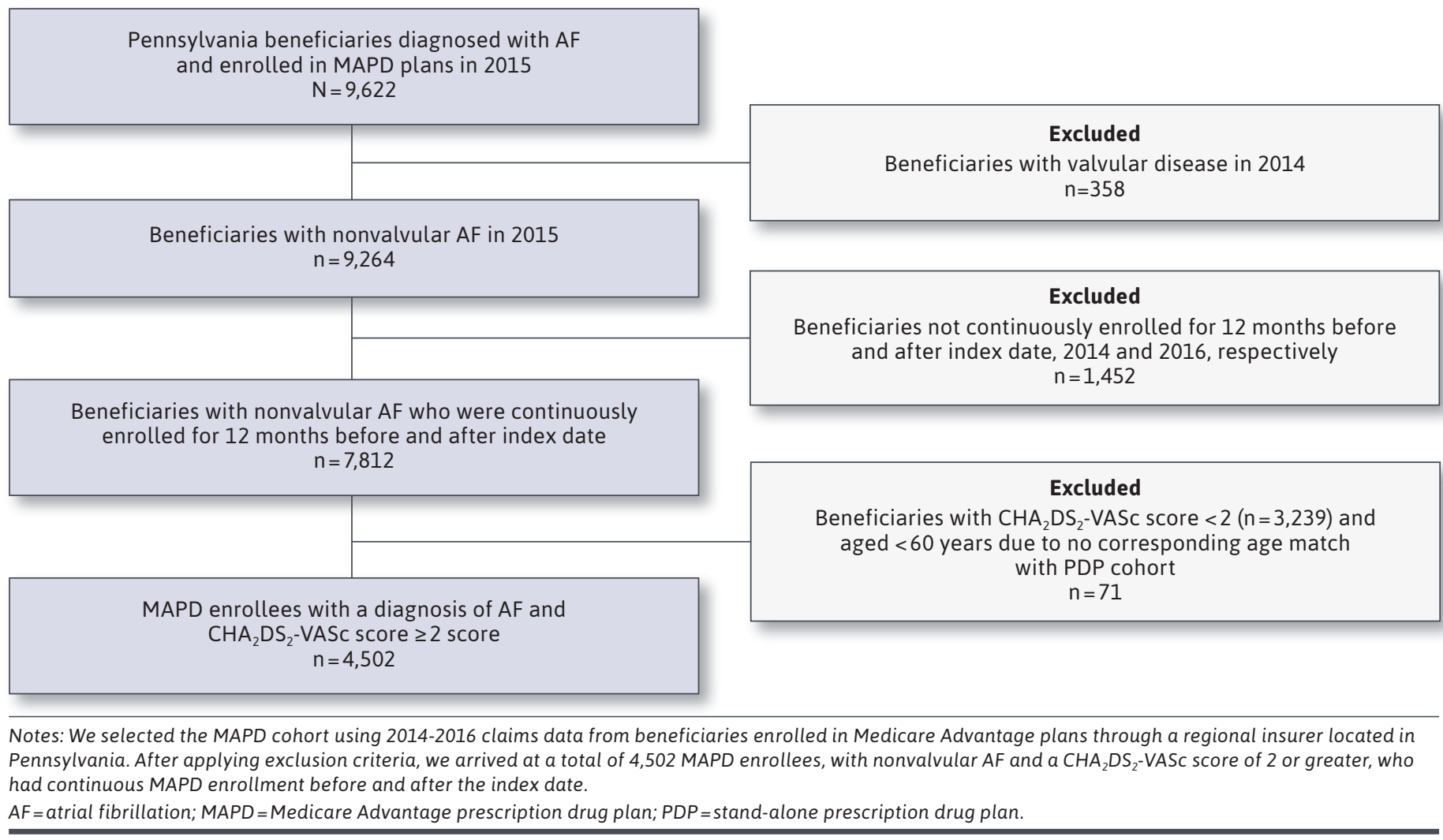

the literature, $\mathrm{OAC}$ adherence was defined as having greater than or equal to $80 \%$ days covered with an OAC. ${ }^{9,16}$ To calculate the number of days covered with an OAC, we extracted all prescriptions for warfarin, apixaban, edoxaban, dabigatran, and rivaroxaban filled after the index date. Then, using dates of fill and days of supply for each prescription, we created a supply diary for 360 days (12 months) after the index date and counted the number of days that each subject had possession of an OAC in the 360 days after the index date.

In line with previous literature, we defined adherent individuals as those who had greater than or equal to 288 ( $80 \%$ of 360 ) days covered, since $80 \%$ is the most commonly used threshold in the literature to define medication adherence. ${ }^{8,9,16-18}$

In secondary analyses, we defined OAC use and adherence separately for warfarin and DOACs. We used the same definitions as in the primary analyses but only used prescription claims for warfarin and DOACs.

\section{STATISTICAL ANALYSES}

We compared baseline covariates between PDP and MAPD cohorts using chi-square tests and 2-sample t-tests as appropriate. Categorical data were reported as frequencies and proportions.

We used a propensity score-matching approach to mitigate confounding. Since younger and healthier individuals tend to be overrepresented in MAPD plans, ${ }^{19}$ propensity score matching can account for the potential imbalances in enrollee characteristics by ensuring that the observed characteristics are similarly distributed across both groups. To calculate the propensity score, we conducted a logistic regression to calculate each beneficiary's propensity of enrolling in a PDP. We used this propensity score and a oneto-one nearest neighbor matching algorithm to generate a 1:1 matched sample.

To ensure that covariates were balanced among matched groups, we calculated standardized differences in covariate means and defined good balance as standardized differences with absolute values less than $0.1{ }^{20}$ Finally, to assess 


\section{TABLE 1 Baseline Characteristics of Study Cohorts Before and After Propensity Score Matching}

\begin{tabular}{|c|c|c|c|c|c|c|}
\hline \multirow[b]{2}{*}{ Variable, n (\%) } & \multicolumn{2}{|c|}{ Before PSM } & \multirow[b]{2}{*}{$P$ value } & \multicolumn{2}{|c|}{ After PSM } & \multirow{2}{*}{$\begin{array}{c}\text { Standardized } \\
\text { difference } \\
\text { in covariate } \\
\text { means }\end{array}$} \\
\hline & $\begin{array}{c}\text { PDP } \\
(n=2,551)\end{array}$ & $\begin{array}{c}\text { MAPD } \\
(n=4,502)\end{array}$ & & $\begin{array}{c}\text { PDP } \\
(n=2,537)\end{array}$ & $\begin{array}{c}\text { MAPD } \\
(n=2,537)\end{array}$ & \\
\hline \multicolumn{7}{|l|}{ Demographics } \\
\hline \multicolumn{7}{|l|}{ Age group, years } \\
\hline$<80$ & 839 (32.89) & $1,977 \quad(43.91)$ & \multirow{2}{*}{$<0.001$} & 839 (33.07) & $906(35.71)$ & \multirow{2}{*}{0.056} \\
\hline$>80$ & $1,712 \quad(67.11)$ & $2,525 \quad(56.09)$ & & $1,698 \quad(66.93)$ & 1,631 (64.29) & \\
\hline Female sex & $1,616 \quad(63.35)$ & $2,160 \quad(47.98)$ & $<0.001$ & $1,602 \quad(63.15)$ & $1,536(60.54)$ & 0.054 \\
\hline \multicolumn{7}{|l|}{ Clinical characteristics } \\
\hline \multicolumn{7}{|l|}{ HAS-BLED score ${ }^{a}$} \\
\hline $0-1$ & $1,305 \quad(51.16)$ & $2,714 \quad(60.28)$ & \multirow{3}{*}{$<0.001$} & $1,305 \quad(51.44)$ & $1,345 \quad(53.02)$ & \multirow{3}{*}{$\mathrm{N} / \mathrm{A}$} \\
\hline $2-3$ & $1,183 \quad(46.37)$ & $1,716(38.12)$ & & $1,173 \quad(46.24)$ & $1,142 \quad(45.01)$ & \\
\hline$\geq 4$ & $63 \quad(2.47)$ & $72 \quad(1.60)$ & & $59 \quad(2.33)$ & $(1.97)$ & \\
\hline $\mathrm{CHF}^{\mathrm{b}}$ & $945 \quad(37.04)$ & $1,341 \quad(29.79)$ & $<0.001$ & 932 (36.74) & 886 (34.92) & 0.038 \\
\hline $\mathrm{HTN}^{\mathrm{b}}$ & $2,295 \quad(89.96)$ & $3,297 \quad(73.23)$ & $<0.001$ & $2,281 \quad(89.91)$ & $2,277 \quad(89.75)$ & 0.005 \\
\hline $\mathrm{MI}^{\mathrm{b}}$ & $79 \quad(3.10)$ & $135 \quad(3.00)$ & 0.817 & $(3.11)$ & $(2.88)$ & 0.014 \\
\hline Renal disease $\mathrm{b}^{\mathrm{b}}$ & $700 \quad(27.44)$ & $1,034 \quad(22.97)$ & $<0.001$ & $692(27.28)$ & $662(26.09)$ & 0.027 \\
\hline Liver disease $^{\mathrm{b}}$ & $45 \quad(1.76)$ & $82 \quad(1.82)$ & 0.862 & $44 \quad(1.73)$ & $(1.42)$ & 0.025 \\
\hline Diabetes $^{\mathrm{b}}$ & $1,076 \quad(42.18)$ & $1,917 \quad(42.58)$ & 0.743 & $1,070(42.18)$ & $1,038 \quad(40.91)$ & 0.026 \\
\hline Stroke or $\mathrm{TIA}^{\mathrm{b}}$ & $618 \quad(24.23)$ & $1,015 \quad(22.55)$ & 0.108 & 608 (23.97) & $560(22.07)$ & 0.044 \\
\hline Intracranial hemorrhage ${ }^{\mathrm{b}}$ & $202 \quad(7.92)$ & $326 \quad(7.24)$ & 0.299 & $(7.88)$ & $(9.07)$ & $\mathrm{N} / \mathrm{A}$ \\
\hline Prior bleeding ${ }^{\mathrm{b}}$ & $683(26.77)$ & $1,005 \quad(22.32)$ & $<0.001$ & $675(26.61)$ & 678 (26.72) & 0.003 \\
\hline \multicolumn{7}{|l|}{ Previous medication use ${ }^{c}$} \\
\hline NSAIDs & $168 \quad(6.59)$ & $(4.62)$ & $<0.001$ & $162 \quad(6.39)$ & $(6.03)$ & 0.014 \\
\hline Anticoagulants & $1,527 \quad(59.86)$ & $2,558 \quad(56.82)$ & 0.013 & $1,517 \quad(59.80)$ & $1,550 \quad(61.10)$ & 0.027 \\
\hline Antiplatelets & $247 \quad(9.68)$ & $454(10.08)$ & 0.588 & $(9.58)$ & $(8.91)$ & 0.023 \\
\hline
\end{tabular}

Note: Data are expressed as $n(\%)$ unless otherwise specified.

a Because Medicare claims data do not contain information on INR levels, we calculated a modified HAS-BLED score that did not include labile INR.

${ }^{b} \mathrm{CHF}, \mathrm{HTN}, \mathrm{MI}$, renal disease, liver disease, diabetes, stroke or TIA, intracranial hemorrhage, and previous bleeding were defined using their respective ICD-9/10-CM codes in the year before the index date.

Medication use was defined in the year before the index date.

$\mathrm{CHF}=$ congestive heart failure; $H T N=$ hypertension; ICD-9/10-CM = International Classification of Diseases, Ninth/Tenth Revision, Clinical Modification;

$I N R=$ international normalized ratio; $M A P D=$ Medicare Advantage prescription drug plan; $M I=$ myocardial infarction; $N / A=$ not applicable; NSAID=nonsteroidal anti-inflammatory drug; OAC = oral anticoagulant; $P D P=$ stand-alone prescription drug plan; $P S M=$ propensity score matching; $T I A=$ transient ischemic attack.

the effect of insurance type on outcomes, we constructed conditional logistic regression models on the matched samples, controlling for covariates specified earlier. All statistical analyses were performed using statistical software SAS version 9.4 (SAS Institute).

\section{SUBGROUP ANALYSES}

We conducted 3 subgroup analyses. In the first analysis, we stratified our sample to beneficiaries who had no fills for any $\mathrm{OAC}$ in the 6 months before the index date. After stratification, we used the same propensity score matching approach as described in the main analysis to compare OAC use and adherence between MAPD and PDP beneficiaries with no history of recent OAC use.

In additional subgroup analyses, we evaluated whether adherence to OACs among OAC users differed between PDP and MAPD beneficiaries. To evaluate this, we conducted subgroup analyses for the outcome of adherence to 


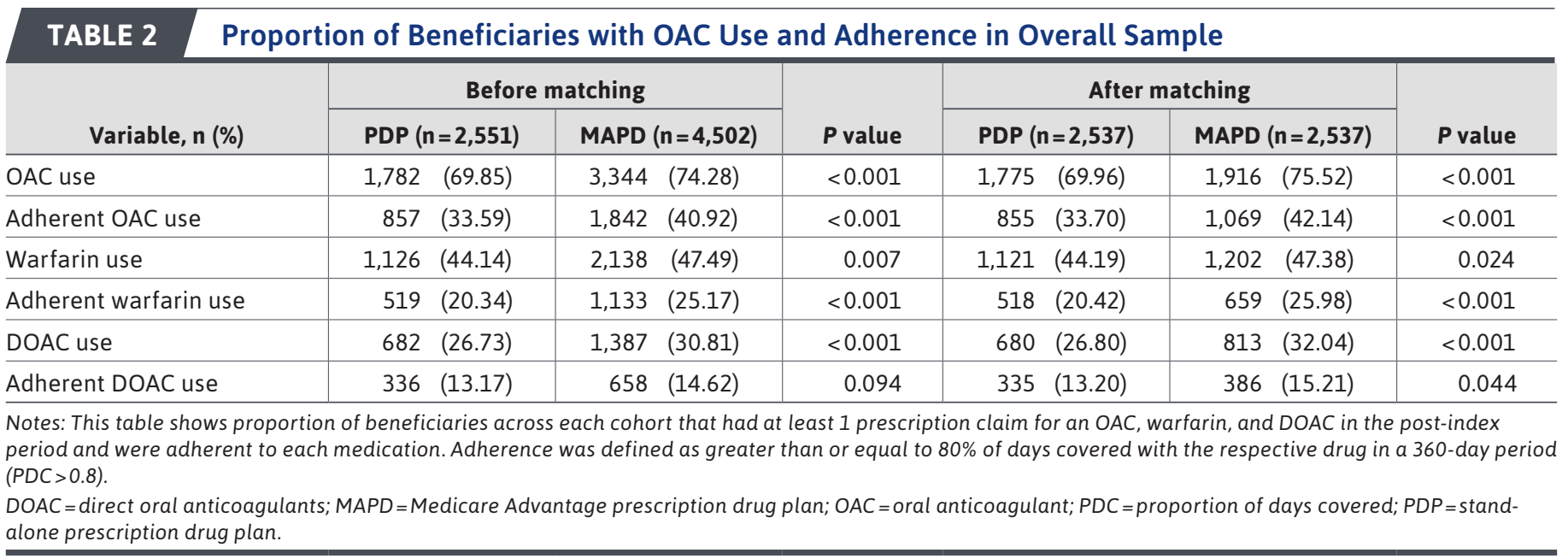

warfarin for patients who filled at least 1 prescription for warfarin after the index date and for the outcome of adherence to DOACs for patients who filled at least 1 prescription for DOACs after the index date.

\section{Results}

\section{PATIENT CHARACTERISTICS}

Our final sample contained 2,551 PDP beneficiaries and 4,502 MAPD beneficiaries who met all inclusion criteria. The propensity score-matched samples included 2,537 beneficiaries in each treatment group.

Table 1 shows the comparison of baseline characteristics before and after propensity score matching across our study sample. Before propensity score matching, the PDP group had a greater proportion of beneficiaries who were older, had higher HAS-BLED scores, and had a history of previous bleeding events and prevalent chronic conditions such as CHF, hypertension, and renal disease compared with beneficiaries in the MAPD group. Additionally, in the PDP group, a greater proportion of beneficiaries used NSAIDs and anticoagulants before the index date. After propensity score matching, we achieved balance for all covariates (standardized difference $<0.10$ ).

\section{UNADJUSTED RESULTS FOR THE OVERALL SAMPLE}

Table 2 shows the proportion of patients across each cohort that had at least 1 fill of an OAC, warfarin, and DOAC and were adherent to each medication before and after propensity score matching. OAC use was higher among MAPD beneficiaries (76\%) than PDP beneficiaries (70\%). Additionally, 42\% of MAPD beneficiaries were adherent to OAC medications compared with $34 \%$ of PDP beneficiaries.

\section{ADJUSTED RESULTS FOR THE OVERALL SAMPLE}

Table 3 shows the adjusted odds ratios (ORs) for use of any OAC, warfarin use, DOAC use, adherence to any OAC, adherence to warfarin, and adherence to DOAC in the overall sample. Enrollment in PDPs was associated with lower odds of OAC use $(\mathrm{OR}=0.67,95 \% \mathrm{CI}=0.56-0.81)$ and adherence $(\mathrm{OR}=0.68,95 \% \mathrm{CI}=0.59-0.78)$ compared with MAPD plans. When observing use and adherence of warfarin, enrollment in PDPs was associated with no difference in warfarin use $(\mathrm{OR}=0.88,95 \% \mathrm{CI}=0.77-1.00)$ but lower odds of adherence to warfarin $(\mathrm{OR}=0.72,95 \% \mathrm{CI}=0.61-0.84)$ compared with MAPD enrollment. Finally, PDP enrollment was associated with lower odds of DOAC use $(\mathrm{OR}=0.79,95 \% \mathrm{CI}=0.69-0.90)$ but no difference in adherence to DOACs (OR=0.88, 95\% $\mathrm{CI}=0.74-1.04)$.

\section{SUBGROUP ANALYSES}

Beneficiaries Without History of OAC Use. After propensity score matching, there were 1,010 beneficiaries in each group who did not use an OAC in the 6 months before the index date. Among them, 39\% used an OAC during the study period in the MAPD cohort, compared with 32\% in the PDP cohort (Supplementary Table 2, available in online article). In adjusted analyses, PDP enrollment was associated with lower odds of $\mathrm{OAC}$ use $(\mathrm{OR}=0.73,95 \% \mathrm{CI}=0.60-0.89)$ and adherence $(\mathrm{OR}=0.69,95 \% \mathrm{CI}=0.51-0.92)$ compared with MAPD enrollment (Supplementary Table 3, available in online article). 
TABLE 3 Adjusted Odds Ratios for OAC Use and Adherence, Overall and by OAC Type

\begin{tabular}{l|cc}
\hline & $\begin{array}{r}\text { Adjusted ORs for PDP vs MAPD } \\
(\mathbf{n}=\mathbf{2 , 5 3 7 )}\end{array}$ \\
\hline Use of OAC & 0.67 & $(0.56-0.81)$ \\
\hline Adherent OAC use & 0.68 & $(0.59-0.78)$ \\
\hline Use of warfarin & 0.88 & $(0.77-1.00)$ \\
\hline Adherent warfarin use & 0.72 & $(0.61-0.84)$ \\
\hline Use of DOACs & 0.79 & $(0.69-0.90)$ \\
\hline Adherent DOAC use & 0.88 & $(0.74-1.04)$ \\
\hline
\end{tabular}

Notes: This table shows the adjusted ORs for use and adherence to any OAC, warfarin, and DOAC, respectively. Use of any OAC, warfarin, and DOAC was defined as having at least 1 fill of the respective drug in the post-index period (after AF diagnosis). Adherent use of OACs, warfarin, and DOACs was defined as greater than or equal to $80 \%$ of days covered with the respective drug in a 360-day period. All analyses were adjusted for covariates specified in the study and compared use and adherence outcomes among PDP beneficiaries and MAPD beneficiaries with MAPD as the reference group.

$A F=$ atrial fibrillation, $D O A C=$ direct oral anticoagulant $; M A P D=$ Medicare Advantage prescription drug plan; $O A C=$ oral anticoagulant; $O R=$ odds ratio; $P D P=$ stand-alone prescription drug plan.

Warfarin and DOAC Users. Among warfarin users, a greater proportion of MAPD beneficiaries were adherent to warfarin compared with PDP beneficiaries in the propensity score-matched sample (60\% vs $47 \%$; Supplementary Table 4, available in online article). Among DOAC users, a slightly greater proportion of PDP beneficiaries were adherent to DOACs as compared with MAPD beneficiaries $(49.3 \%$ vs $49 \%$ ). In adjusted analyses among warfarin users, PDP enrollment was associated with lower odds of adherence to warfarin $(\mathrm{OR}=0.59,95 \% \mathrm{CI}=0.49-0.70$; Supplementary Table 5 , available in online article). In contrast, among DOAC users, there was no difference in the odds of adherent DOAC use between the 2 cohorts $(\mathrm{OR}=1.02,95 \% \mathrm{CI}=0.82-1.28)$.

\section{Discussion}

In this retrospective cohort study, we examined how OAC use and adherence differed between Medicare beneficiaries enrolled in PDPs and MAPD plans. Based on our adjusted analyses on a propensity score-matched cohort, we found that compared with MAPD plans, enrollment in PDPs was associated with $33 \%$ lower odds of OAC use and $32 \%$ lower odds of OAC adherence.

Previous literature has shown that, across data sets and regions, half of $\mathrm{AF}$ patients who are recommended for OACs according to guidelines actually receive it. . $^{8,921,22}$ In our study, we noted that greater than $70 \%$ of Medicare beneficiaries with an indication to receive an OAC received at least 1 prescription claim for an OAC in the study period. Previous research has evaluated how type of Part D coverage affects use and adherence to medications other than OACs. Jung et al found that enrollment in MAPD plans was associated with $2 \%$ higher adherence to statins compared with PDP enrollees, but this difference was determined to be clinically negligible. ${ }^{13}$

The Jung et al study evaluated data from 2007 and reflected the early years of Medicare Part D. Since implementation, Medicare Part D has grown to adopt strategies and infrastructure to enhance medication adherence and other outcomes-focused metrics. Our data contain more recent years and may reflect these changes over the years, which may explain the larger differences detected. Using Medicare claims data from 2007, Erten et al found that MAPD enrollment was associated with increased use of guideline-recommended antidiabetic therapy but lower adherence compared with PDPs. ${ }^{12}$ Our study is an important contribution to this existing literature because it used more recent data and focused on a therapeutic class where continued adherence is crucial for success of therapy.

Our findings support our hypothesis that beneficiaries enrolled in MAPD plans have higher use of and adherence to OACs compared with PDP beneficiaries. Although we are unable to discern the exact cause of the higher OAC use and adherence observed among MAPD beneficiaries, clinical pharmacists in the MAPD plan in our study have a systematic way to calculate $\mathrm{CHA}_{2} \mathrm{DS}_{2}$-VASc scores for patients not on anticoagulants and make recommendations (as appropriate) to clinicians caring for those patients. They also reach out to nonadherent patients. Thus, our findings support the economic model proposed by Goldman et al, which suggests that an integrated insurer that provides substitutable services can maximize use of one service in order to decrease the use of the other substitutable service. ${ }^{13}$

In the context of OAC use for stroke prevention, MAPD plans, as integrated insurers, can achieve optimal use of OACs in order to offset the use of services and resources associated with stroke care. It should be noted that copayments for OACs differ between Part D plans, which could affect the use and adherence to OACs.

The results of our study carry important implications for policy, payer, and patient stakeholders to consider how insurance designs can affect medication use and, subsequently, health outcomes. MAPD plans are incentivized to implement coverage design, infrastructure, and clinical programs that improve OAC use and adherence because of the benefit of averted stroke events and costs. To further evaluate this mechanism, future research needs 
to evaluate the specific features of benefit design or care coordination within these plans that may lead to improved adherence outcomes. Additionally, future research should evaluate how clinical outcomes, such as stroke events or hospitalizations related to AF, differ between MAPD and PDP beneficiaries.

\section{LIMITATIONS}

This study is subject to some limitations. First, claims data only contain information on the filling of prescriptions but not on whether medications were taken as prescribed. Additionally, free samples of warfarin purchased through \$4 generic programs are not captured in claims data, which could lead to an underestimation of OAC use and adherence in our study.

Second, although we collected a comprehensive set of covariates, we did not have information on some factors that can affect adherence, such as cognitive function. Third, this study included data from 2014-2016. Over the years, uptake of DOAC agents has increased due to development of reversal agents, increased clinician comfort and familiarity with DOACs, better safety profile, and guideline changes that recommend DOACs over warfarin for stroke prevention. ${ }^{13,23}$ As a result, a continuation of this study with more recent years of data could provide updated information.

Fourth, although we attempted to control for bias through propensity score matching and adjusted analyses, there may be residual confounding, since PDP patients generally represent a sicker and older population, who may be less likely to be prescribed OACs due to aversion to bleeding risk. ${ }^{24}$ In the same vein, we may have excluded individuals who died within 12 months of the index data due to our inclusion of individuals who were continuously enrolled for 12 months after the index date. This decreased the generalizability of our findings. In line with this, we only observed beneficiaries within Pennsylvania, which limits our generalizability to other geographic locations.

Finally, the use of a single insurer to capture MAPD data may not be generalizable across all MAPD plans, since it only represents a single health plan.

\section{Conclusions}

Using data from Medicare beneficiaries with $\mathrm{AF}$, we found that enrollment in PDPs was associated with 33\% lower odds of OAC use and 32\% lower odds of OAC adherence compared with MAPD enrollment. Given the significant public health burden of AF, the mitigation of stroke risk through the use of OACs is critical in the management of AF to reduce the total clinical and economic burden of disease. Our results suggest that insurance plans that carve-in pharmacy benefits may have an important role to play in improving the use and adherence to OACs among AF patients.

\section{DISCLOSURES}

No outside funding supported this study. Hernandez has received personal fees from BMS and Pfizer, unrelated to this study. The other authors have nothing to disclose.

\section{REFERENCES}

1. Go AS, Mozaffarian D, Roger VL, et al. Heart disease and stroke statistics-2014 update: a report from the American Heart Association. Circulation. 2014;129(3):e28-e292.

2. Go AS, Hylek EM, Phillips KA, et al. Prevalence of diagnosed atrial fibrillation in adults: national implications for rhythm management and stroke prevention: the AnTicoagulation and Risk Factors In Atrial Fibrillation (ATRIA) Study. JAMA. 2001;285(18):2370-75.
3. Miyasaka Y, Barnes ME, Gersh BJ, et al. Secular trends in incidence of atrial fibrillation in Olmsted County, Minnesota, 1980 to 2000 , and implications on the projections for future prevalence. Circulation. 2006;114(2):119-25.

4. Zulkifly H, Lip GYH, Lane DA. Epidemiology of atrial fibrillation. Int J Clin Pract. 2018;72(3):e13070.

5. Newman TV, Chen N, He M, Saba S, Hernandez I. Effectiveness and safety of restarting oral anticoagulation in patients with atrial fibrillation after an intracranial hemorrhage: analysis of Medicare Part D claims data from 2010-2016. Am J Cardiovasc Drugs. 2020;20(5):471-79.

6. Hart RG, Pearce LA, Aguilar MI. Meta-analysis: antithrombotic therapy to prevent stroke in patients who have nonvalvular atrial fibrillation. Ann Intern Med. 2007;146(12):857-67.

7. Verheugt FW, Granger CB. Oral anticoagulants for stroke prevention in atrial fibrillation: current status, special situations, and unmet needs. Lancet. 2015;386(9990):303-10.

8. Lowres N, Giskes K, Hespe C, Freedman B. Reducing stroke risk in atrial fibrillation: adherence to guidelines has improved, but patient persistence with anticoagulant therapy remains suboptimal. Korean Circ J. 2019;49(10):883-907.

9. Hernandez I, He M, Chen N, Brooks MM, Saba S, Gellad WF. Trajectories of oral anticoagulation adherence among medicare beneficiaries newly diagnosed with atrial fibrillation. J Am Heart Assoc. 2019;8(12):e011427.

10. Peterson ED, Pokorney SD. New treatment options fail to close the anticoagulation gap in atrial fibrillation. $\mathrm{J}$ Am Coll Cardiol. 2017;69(20):2485-87.

11. Hess PL, Mirro MJ, Diener HC, et al. Addressing barriers to optimal oral anticoagulation use and persistence among patients with atrial fibrillation: Proceedings, Washington, DC, December 3-4, 2012. Am Heart J. 2014;168(3):239-47.e1. 
12. Erten MZ, Stuart B, Davidoff AJ, Shoemaker JS, Bryant-Comstock L, Shenolikar R. How does drug treatment for diabetes compare between Medicare Advantage prescription drug plans (MAPDs) and stand-alone prescription drug plans (PDPs)? Health Serv Res. 2013;48(3):1057-15.

13. Jung K, McBean AM, Kim J-A. Comparison of statin adherence among beneficiaries in MA-PD plans versus PDPs. J Manag Care Pharm. 2012;18(2):106-15. doi:10.18553/jmcp.2012.18.2.106

14. January CT, Wann LS, Alpert JS, et al. 2014 AHA/ACC/HRS guideline for the management of patients with atrial fibrillation: a report of the American College of Cardiology/American Heart Association Task Force on Practice Guidelines and the Heart Rhythm Society. J Am Coll Cardiol. 2014;64(21):e1-e76.

15. January CT, Wann LS, Alpert JS, et al. 2014 AHA/ACC/HRS guideline for the management of patients with atrial fibrillation. Circulation. 2014;130(23):e199-e267.
16. Hernandez I, He M, Brooks MM, Saba S, Gellad WF. Adherence to anticoagulation and risk of stroke among medicare beneficiaries newly diagnosed with atrial fibrillation. Am J Cardiovasc Drugs. 2020;20(2):199-207.

17. Sørensen R, Jamie Nielsen B, Langtved Pallisgaard J, Ji-Young Lee C, Torp-Pedersen C. Adherence with oral anticoagulation in non-valvular atrial fibrillation: a comparison of vitamin $\mathrm{K}$ antagonists and non-vitamin $\mathrm{K}$ antagonists. Eur Heart J Cardiovasc Pharmacother. 2017;3(3):151-56.

18. Karve S, Cleves MA, Helm M, Hudson TJ, West DS, Martin BC. Good and poor adherence: optimal cut-point for adherence measures using administrative claims data. Curr Med Res Opin. 2009;25(9):2303-10.

19. Jacobson G, Neuman T, Freed M, Damico A. What percent of new Medicare beneficiaries are enrolling in Medicare Advantage? June 6, 2019. Accessed March 19, 2020. https://www.kff.org/medicare/ issue-brief/what-percent-of-new-medicare-beneficiaries-are-enrolling-in-medicare-advantage/
20. Cohen J. Statistical power analysis. Curr Dir Psychol Sci. 1992;1(3):98-101.

21. Baik SH, Hernandez I, Zhang Y. Evaluating the initiation of novel oral anticoagulants in Medicare beneficiaries. J Manag Care Spec Pharm. 2016;22(3):28192. doi:10.18553/jmcp.2016.22.3.281

22. Lubitz SA, Khurshid S, Weng L-C, et al. Predictors of oral anticoagulant non-prescription in patients with atrial fibrillation and elevated stroke risk. Am Heart J. 2018;200:24-31.

23. January CT, Wann LS, Calkins H, et al. 2019 AHA/ACC/HRS focused update of the 2014 AHA/ACC/HRS guideline for the management of patients with atrial fibrillation: a report of the American College of Cardiology/American Heart Association Task Force on Clinical Practice Guidelines and the Heart Rhythm Society. J Am Coll Cardiol. 2019;74(1):104-32.

24. Elliott MN, Landon BE, Zaslavsky AM, et al. Medicare prescription drug plan enrollees report less positive experiences than their Medicare Advantage counterparts. Health Aff (Millwood). 2016;35(3):456-63. 